\title{
Designing the Layout Structure of Manual Order Picking Areas in Warehouses
}

\author{
KEES JAN ROODBERGEN ${ }^{1, *}$, GUNTER P. SHARP 2 and IRIS F.A. VIS ${ }^{3}$ \\ ${ }^{1}$ RSM Erasmus University, P.O. box 1738, 3000 DR Rotterdam, The Netherlands \\ ${ }^{2}$ Georgia Institute of Technology, School of Industrial and Systems Engineering, Atlanta, GA. \\ 3 VU University Amsterdam, Faculty of Economics and Business Administration, De Boelelaan \\ 1105, Room 3A-31, 1081 HV Amsterdam, The Netherlands
}

Please refer to this article as:

Roodbergen, K.J., Sharp, G.P., and Vis, I.F.A. (2008), Designing the layout structure of manual order picking areas in warehouses. IIE Transactions 40(11), 1032-1045.

\begin{abstract}
Order picking is the warehousing process by which products are retrieved from their storage locations in response to customers' orders. Its efficiency can be influenced through the layout of the area and the operating policies. We present a model that minimizes travel distances in the picking area by identifying an appropriate layout structure consisting of one or more blocks of parallel aisles. The model has been developed for one commonly used routing policy, but it is shown to be fairly accurate for some other routing policies as well.
\end{abstract}

\section{Introduction}

A warehouse typically consists of various areas, including shipping and receiving areas, bulk storage and order picking areas. A good overview of warehouse operations is given in Gu et al. (2007). Due to several trends, among which the emergence of e-commerce, there is an increasing emphasis on the order picking operation, which consists of retrieving individual items from storage on the

\footnotetext{
${ }^{*}$ Corresponding author
} 
basis of customers' orders. This order picking process is often one of the most laborious and costly activities in a warehouse (Tompkins et al., 2003).

A typical design project for an order picking area starts by identifying the required size of the area, the appropriate racking (for example, flow racks, pallet racks or shelves) and the equipment (for example, order picking trucks or picking carts). Next, the layout structure of the area is to be determined. Finally, operating policies are chosen to control the order picking process concerning, for example, assignment of products to storage locations and sequencing of items on the pick list. This sequential approach is convenient in practice, but does not necessarily lead to the best possible solution. In this paper, we present a 'reverse' method that can optimize the layout for the order picking area based on properties of the operating policies. A statistical estimate for the average travel distance is derived and serves as the objective function in the layout optimization problem. Compared to existing methods our approach allows more layout variations, specifically the number of blocks is not limited (see Section 2). Similar results could also be obtained by means of a massive simulation study. Our analytical model is, however, preferred over simulation when considering future applicability in practice. This holds mainly because analytical formulas are easier to incorporate in spreadsheet applications since they are more compact, simpler to implement, less calculation intensive, and require less memory storage than simulation code. Simulation will therefore only be used in this paper to validate our analytical model.

In Section 2 we describe the order picking area and the layout optimization problem. Routing methods will be treated in Section 3. The objective function of the model is derived in Section 4. The quality of the model is evaluated in Section 5. Some implications for new warehouse designs are given in Section 6 and Section 7 gives concluding remarks. Finally, a layout optimization example is presented in the Appendix. 


\section{Layout structure}

We consider a manual order picking operation, where order pickers walk or drive through a warehouse to retrieve products from storage. Picked items are placed on a pick device, which the order picker takes with him on his route. Figure 1 shows various aspects of the layout of an order picking area. The layout structure is composed of several pick aisles, which have racks on both sides to store products in. Order pickers can change from one pick aisle to another pick aisle at one of the cross aisles, which are positioned perpendicular to the pick aisles. Typically, there are at least two cross aisles, one in the front and one in the back. More cross aisles in between the front and back cross aisles can be added to increase the number of possibilities to change aisles. Cross aisles do not contain pick locations. The part of a pick aisle that is between two adjacent cross aisles, is referred to as a subaisle. All subaisles between two cross aisles taken together are called a block. The main advantage of having extra cross aisles in a warehouse consists of the increased routing options, which may result in lower travel distances (Vaughan and Petersen, 1999). On the other hand, warehouse size must increase if more cross aisles are added, because total storage space must be kept constant to meet predefined requirements.

\section{XXXXXXXXXXXXXX}

\section{Insert figure 1}

\section{XXXXXXXXXXXXXX}

Several methods exist to control the order picking process, including but not limited to storage assignment policies and routing policies. Storage assignment policies are used in a warehouse to determine which product is to be positioned at which location. Numerous rules exist for storage assignment ranging from random storage to policies that arrange products based on their demand frequency (Gu et al., 2007). For our model, item locations are assumed to be determined randomly according to an uniform distribution. Clearly, activity-based item location could possibly ask for a different layout structure. We will consider only random storage assignment since this strategy 
can be considered as a base-line against which layouts with activity-based storage assignment can be compared. Furthermore, random storage is a commonly used storage strategy. For example, for some e-commerce products demand frequency may be too volatile, product life too short, or the required storage location reorganizations too costly to maintain a storage policy based on demand frequencies.

Routing policies are used to sequence the items on the pick list to reduce travel times. Several heuristics and optimal algorithms exist for various situations (Hall, 1993). Order pickers are assumed to be able to traverse an aisle in either direction and to change direction within an aisle. The path of the order picker is considered to follow the exact middle of the aisles. Any distance between a pick location and the middle of the aisle is assumed to be negligible compared to the total travel distance. In practice, aisle width is typically dictated by equipment requirements and expected risk of congestion. Therefore, we do not consider the aisle width as a variable in the layout optimization. See Gue et al. (2006) for issues related to congestion. Every item can be picked from the rack by the order picker without climbing or using a lifting device, which implies that warehouse height is not a factor in this research. Picked orders have to be deposited at the depot, where the picker also receives the instructions for the next route.

Several papers address the problem of designing order picking areas. Petersen (1997) studied interactions between routing policies and layout for the order picking area by means of simulation. However, that paper does not provide a general design methodology. Hall (1993) studied the impact of layout on order picking efficiency through analytical estimates for travel distance in one-block layouts. Roodbergen and Vis (2006) developed a model that is capable of finding the best layout structure for the order picking area. Their analysis shows the possibilities and impact of developing a layout based on characteristics of the operating policies. The results are, however, limited by the fact that they allow only layouts consisting of one block in their analysis. Caron et al. (2000) and Le-Duc and De Koster (2005) study warehouses consisting of two blocks with the depot located 
between the two blocks at the head of the middle cross aisle. The model we present in this paper is capable of selecting the best option from layouts with any number of aisles and any number of blocks.

For manual order picking, average travel distance is influenced by four major factors: (1) the length of the pick aisles, (2) the number of pick aisles, (3) the number of blocks, and (4) the number of picks per route. The basic model presented here optimizes the layout structure for a fixed number of picks, however, this constraint will be relaxed at a later stage. Actually, the location of the depot can be considered a fifth factor. We will, however, assume that the depot is always located at the head of the left-most pick aisle. We make this assumption because the impact of the depot location on travel distances is usually not very large. Petersen (1997) found that the influence of the depot location on travel distances is less than $6 \%$ and falls below $1 \%$ if there are 15 or more picks per route. The effect of moving the depot from the front cross aisle to the side of the warehouse (as indicated in Figure 1 by 'alternative depot location') is more difficult to predict and therefore investigated explicitly in Section 5 .

We define the following variables:

$n$ number of pick aisles (integer),

$k$ number of blocks (integer),

$y$ length of a pick aisle along the pick face (i.e., the length of a pick aisle excluding the width of the cross aisles).

We define the following parameters:

$S$ total aisle length, measured along the pick face,

$m$ the number of picks (integer),

$w_{c}$ width of a cross aisle, 
$w_{a}$ center-to-center distance between two adjacent pick aisles.

See Figure 1 for a graphical illustration of $y, w_{a}$ and $w_{c}$.

Expanding the one-block optimization model of Roodbergen and Vis (2006) for multiple blocks, we can formulate the problem as follows:

$$
\begin{array}{ll} 
& \min T_{m}(n, k, y) \\
\text { s.t. } & n \cdot y=S \\
& n \geq 1, k \geq 1 \\
& n, k \text { integer }
\end{array}
$$

The minimization of the travel distance $T_{m}(n, k, y)$ is to be performed under the condition that total storage space is kept constant. This restriction is modeled as $n \cdot y=S$. That is, total aisle length along the pick face is constant. In Section 4 we derive an expression for the average travel distance $T_{m}(n, k, y)$ for the case where order pickers travel through the warehouse according to the S-shape heuristic. The routing heuristic itself is described in the next section. To find the optimum, we will use complete enumeration over all reasonable values of the model's variables. From the experiments presented in this paper, it has appeared this can be done in less than one minute. Therefore, this is no practical impediment for applying the model.

\section{Routing of order pickers}

Many methods exist to route an order picker through a warehouse. Ratliff and Rosenthal (1983) developed an efficient algorithm based on dynamic programming, which can find a shortest route for warehouses consisting of one block. Hall (1993) describes several heuristics for routing order pickers in the same layout. Vaughan and Petersen (1999) present a heuristic to route order pickers in a warehouse with multiple blocks. Their approach assumes that order pickers visit each pick aisle just once in a fixed sequence from left to right. The cross aisles used to make connections between 
the pick aisles are optimized. Adaptations of the heuristics of Hall (1993) for multiple-block layouts are presented in Roodbergen and De Koster (2001), along with a new routing heuristic.

The S-shape heuristic is one of the few heuristics from literature that is also widely used in practice. The adapted version for multiple-block situations, which we use here, was described in Roodbergen and De Koster (2001). Basically, any subaisle containing at least one pick location is traversed through the entire length. Subaisles where nothing has to be picked are skipped. In the following more elaborate description of the heuristic, letters between brackets correspond to the letters in the example route depicted in Figure 2. In Section 5 we will evaluate to what extent the layouts determined with the model of Section 2 are sensitive to changes in the routing method.

\section{$\operatorname{XXXXXXXXXXXXXX~}$}

Insert figure 2

\section{XXXXXXXXXXXXXX}

\section{S-shape heuristic}

1. Determine the left most pick aisle that contains at least one pick location (called left pick aisle) and determine the block farthest from the depot that contains at least one pick location (called farthest block).

2. The route starts by going from the depot to the front of the left pick aisle (a).

3. Traverse the left pick aisle up to the front cross aisle of the farthest block (b).

4. Go to the right through the front cross aisle of the farthest block until a subaisle with a pick is reached (c). If this is the only subaisle in this block with pick locations then pick all items and return to the front cross aisle of this block. If there are two or more subaisles with picks in this block, then entirely traverse the subaisle (d). Continue with step 5.

5. At this point, the order picker is in the back cross aisle of a block, call this block the current 
block. There are two possibilities.

(1) There are picks remaining in the current block (not picked in any previous step). Determine the distance from the current position to the left-most subaisle and the right-most subaisle of this block with picks (which have not been retrieved before). Go to the closer of these two

(e). Entirely traverse this subaisle (f). Continue with step 6 .

(2) There are no items left in the current block that have to be picked. In this case, continue in the same pick aisle (i.e., the last pick aisle that was visited in either step 7 or in this step) to get to the next cross aisle $(\mathrm{g})$ and continue with step 8 .

6. If there are items left in the current block that have to be picked, then traverse the cross aisle towards the next subaisle with a pick location (h) and entirely traverse that subaisle (j). Repeat this step until there is exactly one subaisle left with pick locations in the current block. Continue with step 7.

7. Go to the last subaisle with pick locations of the current block (k). Retrieve the items from the last subaisle and go to the front cross aisle of the current block (m). This step can actually result in two different ways of traveling through the subaisle (1) entirely traversing the subaisle or (2) entering and leaving the subaisle from the same side.

8. If the block closest to the depot has not yet been examined, then return to step 5 .

9. Finally, return to the depot (n).

\section{Average travel distance estimation}

Research on the subject of travel distance estimation has mainly been restricted to warehouses consisting of a single block, see Chew and Tang (1999), Hall (1993), Jarvis and McDowell (1991), Kunder and Gudehus (1975) and Roodbergen and Vis (2006). Caron et al. (1998) and Le-Duc and De Koster (2005) studied a two-block layout with turnover-based storage policies and with the 
depot at the head of the middle cross aisle. However, for analytical purposes such a layout is in many aspects similar to an one-block layout with the depot at the head of the left-most aisle.

In this section we give a statistical estimate for the average travel distances in a warehouse with any number of blocks when using the S-shape heuristic for routing. We have decomposed the order picking routes into eleven components based on the described elements of the S-shape heuristic and derive travel distance estimates for each component. In Figure 3 we graphically illustrate most of these eleven components with matching numbers. At the end of this section, we present a formulation for the total average travel distance estimate. First, we define the following:

$i$ is used to indicate the left-most pick aisle that contains a pick location. Note that aisles will be numbered from right to left. That is, the right-most aisle of the warehouse is aisle 1 .

$j$ is used to indicate the block farthest from the depot that contains pick locations. Blocks will be numbered from front to back.

\section{$\operatorname{XXXXXXXXXXXXXX~}$}

\section{Insert figure 3}

\section{XXXXXXXXXXXXXX}

\section{Traveling through subaisles with picks, $E^{(1)}$}

First, we give an estimate for the expected travel distance for all subaisles with at least one pick that are traversed. With this estimate we assume that a subaisle with pick is traversed entirely. Estimate $E^{(2)}$ will be used to correct for subaisles that are entered and left from the same side. The estimate $E^{(1)}$ is based on the expected number of subaisles to be visited and the length of each subaisle and given by:

$$
E^{(1)}=n k\left[1-\left(\frac{n k-1}{n k}\right)^{m}\right] \cdot\left(\frac{y}{k}+w_{c}\right)
$$

The expected number of subaisles that have to be visited, can be formulated as $n k\left[1-\left(\frac{n k-1}{n k}\right)^{m}\right]$. In other words, the term between square brackets is 1 minus the probability that a certain subaisle 
does not contain pick locations. A more precise estimate for the number of subaisles to be visited is possible, however, in Kunder and Gudehus (1975) it is shown that an estimate like this is generally adequate. The length of a subaisle is equal to the length of a subaisle along the pick face $(y / k)$ plus two times half the width of a cross aisle $\left(w_{c}\right)$. This is because we assume that the order picker travels exactly through the middle of the aisles and cross aisles.

\section{Correction of travel distance for turns within subaisles, $E^{(2)}$}

Occasionally, aisles are entered and left from the same side. This occurs in any block where the last subaisle is entered from the front (see step 7 of the routing heuristic). First, we need to estimate for each block $k$ the one-way distance to the farthest location in the last subaisle, which can be expressed as part of the length of a subaisle along the pick face $\left(\frac{m /(n k)}{m /(n k)+1} \cdot \frac{y}{k}\right)$. This is based on the known statistical property that the maximum of $b$ continuous uniformly distributed $[0,1]$ variables $\left\{X_{i}\right\}$ is given by $E\left[\max \left\{X_{1}, X_{2}, \ldots, X_{b}\right\}\right]=b /(b+1)$, combined with the fact that the expected number of picks in a subaisle equals $m /(n k)$. Clearly, while making a turn this distance needs to be traversed twice. The distance related to traversing this aisle without making a turn $(y / k)$ as incorporated in $E^{(1)}$ needs to be deducted from $E^{(2)}$ to prevent double counts. The estimate $E^{(2)}$ is formulated as follows:

$$
E^{(2)}= \begin{cases}\frac{2 y m}{m+n k}-y & \text { if } n \text { odd, and } \frac{m}{n k}>1 \\ 0 & \text { otherwise }\end{cases}
$$

which follows from the fact that

$$
k\left(2 \frac{m /(n k)}{m /(n k)+1} \frac{y}{k}-\frac{y}{k}\right)=\frac{2 y m}{m+n k}-y .
$$

Both estimate $E^{(1)}$ and $E^{(2)}$ deal with subaisles containing picks. The routing heuristic sometimes lets an order picker travel through a subaisle that does not contain picks. Next, we give $E^{(3)}$ to handle this situation. 


\section{Traveling through subaisles without picks, $E^{(3)}$}

As explained in Section 3, occasionally some subaisles without pick locations may need to be traversed. Namely, the left-most pick aisle $i$ is traversed entirely to reach the farthest block with picks (see step 3 of the routing heuristic), regardless of the presence of picks in the subaisles that are passed. An order picker may also traverse empty subaisles on his way from the back to the front of the warehouse (step 5.2 of the heuristic). This occurs if there are no picks at all in a block. To include this additional travel we propose the estimate $E^{(3)}$.

$$
E^{(3)}=\left(\frac{y}{k}+w_{c}\right) \sum_{i=1}^{n} \sum_{j=1}^{k} A_{i j} E_{i j}^{(3)}
$$

with

$$
\begin{aligned}
& A_{i j}=\left[\left(\frac{i}{n}\right)^{m}-\left(\frac{i-1}{n}\right)^{m}\right] \cdot\left[\left(\frac{j}{k}\right)^{m}-\left(\frac{j-1}{k}\right)^{m}\right] \\
& E_{i j}^{(3)}=(j-1)\left(\frac{i j-1}{i j}\right)^{m}+(j-1)\left(\frac{i j-i+1}{i j}\right)^{m} .
\end{aligned}
$$

As explained in steps 1 and 2 of the routing heuristic, both the left-most pick aisle $i$ and the farthest block $j$ need to be determined. The probability that $j$ is the farthest block can be expressed as the probability that all picks fall into blocks 1 through $j$ with at least one pick in block $j$, namely $\left[\left(\frac{j}{k}\right)^{m}-\left(\frac{j-1}{k}\right)^{m}\right]$. Similarly, the probability that $i$ is the left-most pick aisle can be seen to equal $\left[\left(\frac{i}{n}\right)^{m}-\left(\frac{i-1}{n}\right)^{m}\right]$. We obtain $A_{i j}$ by combining both probabilities.

The component $E_{i j}^{(3)}$ consists of the number of blocks that must be traversed $(j-1)$, multiplied by the probability that this happens without picking. The probability of traversing a block without picking from front to back equals $((i j-1) / i j)^{m}$. This holds because the probability that one pre-specified pick will not be in one pre-specified subaisle out of the available $i j$ subaisles equals $(i j-1) / i j$. Similarly, subaisles $1, \ldots, i-1$ must all be empty if a block is traversed from back to front without picks, which has a probability of $(i j-i+1) / i j$.

We take the summation over all possible values of $i$ and $j$, the appropriate probability $A_{i j}$ and 
the expected travel distance for each combination of $i$ and $j$, which is the product of $\left(y / k+w_{c}\right)$ and $E_{i j}^{(3)}$, to obtain an estimate of travel distances in subaisles without picks. We have now covered travel distances within the aisles by means of estimates $E^{(1)}, E^{(2)}$, and $E^{(3)}$. Next, we need to add distances traveled in the cross aisles.

\section{Traveling from the depot to the first pick aisle, $E^{(4)}$}

The first distance an order picker travels in one of the cross aisles, is when he goes from the depot (in aisle $n$ ) to the left-most aisle with picks (aisle $i$ ). Similar to $A_{i j}$ we need to calculate the probability that aisle $i$ is the left-most pick aisle. For all possible values of $i$ we take the number of aisles that need to be passed before we reach aisle $i$ while starting in $n$ (which equals $n-i$ ) and multiply it by the width of an aisle $\left(w_{a}\right)$ to obtain the corresponding travel distances. Summarizing, $E^{(4)}$ can be calculated as follows:

$$
E^{(4)}=w_{a} \sum_{i=1}^{n}\left((n-i) \cdot\left[\left(\frac{i}{n}\right)^{m}-\left(\frac{i-1}{n}\right)^{m}\right]\right) .
$$

Besides $E^{(4)}$, the distances traveled in cross aisles can be divided into the following components: distances traveled in cross aisles of the farthest block that contains picks $E^{(5)}$, distances traveled in the other blocks to connect subaisles that contain picks $E^{(6)}$ and distances traveled to go from one block to the next $E^{(7)}$. The remaining travel distances in cross aisles consist of returning to the depot after the last pick, which will be treated later on.

\section{Traveling in the cross aisles of the farthest block, $E^{(5)}$}

After arriving at the front cross aisle of the farthest block that contains picks (block $j$ ), the order picker travels through this block's front cross aisle until a subaisle with picks is reached (see step 4 of the routing heuristic). Thereafter, all subaisles with picks are handled until the order picker ends at the right-most subaisle with picks. Let $g$ be the right-most subaisle with picks (with $g=1$ indicating the right-most subaisle) in block $j$, then the distance traveled in the cross aisles of block 
$j$ equals $(i-g) w_{a}$. This information can be used to estimate the distance traveled in the cross aisles of block $j$ as follows:

$$
E^{(5)}=w_{a} \sum_{i=2}^{n} \sum_{j=1}^{k} A_{i j} E_{i j}^{(5)}
$$

where

$$
E_{i j}^{(5)}=\sum_{g=1}^{i-1}\left\{(i-g) \sum_{u=1}^{m} B\left(u, m, \frac{i-1}{i j}\right)\left[\left(\frac{i-g}{i-1}\right)^{u}-\left(\frac{i-g-1}{i-1}\right)^{u}\right]\right\}
$$

with the binomial distribution $B$ given by:

$$
B(u, m, p)=\left(\begin{array}{c}
m \\
u
\end{array}\right)(p)^{u}(1-p)^{m-u}
$$

The probability that $g$ is the right-most subaisle with picks in block $j$ given that $i$ is the leftmost pick aisle is given by the term between square brackets. The binomial distribution gives the probability that there are exactly $u$ picks in subaisles $1, \ldots, i-1$.

\section{Traveling in the cross aisles while picking, $E^{(6)}$}

Next we consider the estimate for the distance traveled in cross aisles of blocks other than block $j$ while picking items. That is, we estimate the cross aisle travel resulting from steps 6 and 7 of the routing heuristic as follows:

$$
E^{(6)}=w_{a} \sum_{i=3}^{n} \sum_{j=1}^{k} A_{i j} E_{i j}^{(6)}
$$

where

$$
E_{i j}^{(6)}=(j-1) \sum_{u=1}^{m-1}\left\{B\left(u, m, \frac{i-1}{i j}\right) \cdot \sum_{\ell=1}^{i-1}[\ell \cdot(i-1-\ell) \cdot Q(\ell)]\right\},
$$

where the probability that all picks fall in $\ell+1$ consecutive subaisles is given by:

$$
Q(\ell)=\left(\frac{\ell+1}{i-1}\right)^{u}-2\left(\frac{\ell}{i-1}\right)^{u}+\left(\frac{\ell-1}{i-1}\right)^{u}
$$

$Q(\ell)$ gives the probability that all items of this area fall in subaisles $\ell_{1}, \ldots, \ell_{2}$, at least one pick falls in $\ell_{1}$, at least one pick falls in $\ell_{2}$ and $\ell_{2}-\ell_{1}=\ell$. There are $(i-1-\ell)$ different combinations of $\ell+1$ consecutive subaisles possible from a set of $i-1$ subaisles. The distance traveled is $w_{a} \cdot \ell$. 
Furthermore, we take the sum over all possible values for the number of picks $(u)$ in this area and multiply by the corresponding probabilities. We consider all blocks except for the farthest block $j$, which has been dealt with in $E^{(5)}$. As a result, we multiply by $(j-1)$.

\section{Traveling in the cross aisles to connect blocks, $E^{(7)}$}

We need a factor to account for the expected travel from the last visited subaisle of the previous block to the first subaisle to be visited in the next block as described in step 5.1 of the heuristic. We have $E_{i j}^{(6)}$ as an estimate for the distance between the left and right-most subaisle in a block. The distance between the subaisles 1 and $i-1$ is equal to $i-2$. Therefore, the end point of the previous block can vary over a distance of $w_{a}(i-2)-E_{i j}^{(6)}$. The start point of the next block and end point of the previous block are uniformly distributed over this distance. Treating aisle locations as continuous random variables, an approximation for the expected distance between the location of the two points is $\frac{1}{3} \cdot\left((i-2) \cdot w_{a}-E_{i j}^{(6)}\right)$. This distance only needs to be traveled if there is at least one pick in the next block. Otherwise, the order picker just traverses the nearest subaisle to go straight to the next block. We do this for the $(j-1)$ connections between the $j$ blocks that are visited. Therefore, $E^{(7)}$ is given by:

$$
E^{(7)}=\sum_{i=2}^{n} \sum_{j=1}^{k} A_{i j} E_{i j}^{(7)}
$$

with

$$
E_{i j}^{(7)}=(j-1) \cdot \frac{1}{3} \cdot\left((i-2) \cdot w_{a}-E_{i j}^{(6)}\right) \cdot\left(1-\left(\frac{i j-i+1}{i j}\right)^{m}\right)
$$

With estimates $E^{(4)}-E^{(7)}$ we have covered all travel distances in cross aisles, except for the distance to return to the depot after the last pick. This distance depends on the total number of blocks with pick locations and estimates for this are given in the next two subsections. 


\section{Traveling back to the depot if the number of visited blocks is even, $E^{(8)}$}

Consider the sequence in which subaisles of a block will be visited. The route starts by going to the farthest block $j$ and the subaisles in this block will be visited from left to right (see Figure 3 ). Then in the next block with picks, subaisles can be visited from right to left. In the third block with picks, subaisles are visited left to right, and so on. If the number of visited blocks is even, then it is most likely that the order picker finishes picking at the left of the front cross aisle. Note that we consider the number of visited blocks, not the total number of blocks in the layout. The travel distance in the front cross aisle of the warehouse, if an even number of blocks has been visited, is given by:

$$
E^{(8)}=w_{a} \cdot \sum_{i=1}^{n} \sum_{j=2}^{k} p_{j} A_{i j} E_{i j}^{(8)}
$$

with

$$
E_{i j}^{(8)}=\sum_{g=1}^{i-1} \sum_{u=1}^{m-1}\left(B\left(u, m, \frac{i-1}{i j}\right) \cdot(n-g) \cdot\left[\left(\frac{g}{i-1}\right)^{u}-\left(\frac{g-1}{i-1}\right)^{u}\right]\right),
$$

where $B\left(u, m, \frac{i-1}{i j}\right)$ gives the probability that there are exactly $u$ picks in block 1 ; the probability that the left-most subaisle visited in block 1 equals subaisle $g$ is given by $\left[\left(\frac{g}{i-1}\right)^{u}-\left(\frac{g-1}{i-1}\right)^{u}\right]$; and $w_{a}(n-g)$ is the distance from subaisle $g$ to the depot.

The probability that the number of blocks to be visited is even, is given by:

$$
p_{j}=\sum_{h \in H}\left(\begin{array}{l}
j \\
h
\end{array}\right)\left(\frac{h}{j}\right)^{m} \cdot\left[1-\sum_{\gamma=1}^{h-1}(-1)^{\gamma+1}\left(\begin{array}{c}
h \\
h-\gamma
\end{array}\right)\left(\frac{h-\gamma}{h}\right)^{m}\right]
$$

with $H=\{h \mid 1 \leq h \leq k, h \leq m$ and $h$ is even $\}$.

This is based on the inclusion-exclusion rule. To briefly explain this, consider the following. Suppose the farthest block is block $j$. The probability that all items are in at most $h$ of $j$ blocks equals $(h / j)^{m}$ multiplied by the number of possibilities to select $h$ blocks from $j$ blocks, $\left(\begin{array}{l}j \\ h\end{array}\right)$. Next we need to find the probability of all picks being in exactly $h$ of $j$ blocks. To obtain this, we start with a (conditional) probability of 1 and subtract the probability that all picks are in $h-1$ blocks, given that they are in 
at most $h$ blocks: $\left(\begin{array}{c}h \\ h-1\end{array}\right)\left(\frac{h-1}{h}\right)^{m}$. But now we subtracted the probability that all picks are in $h-2$ blocks too often, so we need to add $\left(\begin{array}{c}h \\ h-2\end{array}\right)\left(\frac{h-2}{h}\right)^{m}$ to compensate. And so on.

\section{Traveling back to the depot if the number of visited blocks is odd, $E^{(9)}$}

If the number of visited blocks is odd, the order picker most likely ends his route at the right-most subaisle of the block closest to the depot. Similar to $E^{(8)}$, we can formulate this as:

$$
E^{(9)}=w_{a} \cdot \sum_{i=1}^{n} \sum_{j=2}^{k}\left(1-p_{j}\right) A_{i j} E_{i j}^{(9)}
$$

with

$$
E_{i j}^{(9)}=\sum_{g=1}^{i-1} \sum_{u=1}^{m-1}\left(B\left(u, m, \frac{i-1}{i j}\right) \cdot(n-g) \cdot\left[\left(\frac{i-g}{i-1}\right)^{u}-\left(\frac{i-g-1}{i-1}\right)^{u}\right]\right) .
$$

Both $E^{(8)}$ and $E^{(9)}$ do not include the distance that has to be traveled through the front cross aisle if the block closest to the depot has no picks. Therefore, we derive estimate $E^{(10)}$.

\section{Traveling back to the depot if there are no picks in block $1, E^{(10)}$}

With estimate $E^{(10)}$ we estimate the distance traveled in the front cross aisle if the last pick of the route has been collected before reaching block 1 . In this case, we assume that the order picker has to travel half the length of the front cross aisle to return to the depot. As a result, $E^{(10)}$ can be estimated as:

$$
E^{(10)}=w_{a} \sum_{i=1}^{n} \sum_{j=1}^{k} A_{i j}\left(n-\frac{i}{2}\right)\left(\frac{i j-i+1}{i j}\right)^{m}
$$

\section{Traveling back to the depot if all picks are in one block, $E^{(11)}$}

Finally, we need to determine the distance the order picker travels if all picks are in the block closest to the depot. That is, if $j=1$. Similar to the previous estimates, this estimate is given by:

$$
E^{(11)}=w_{a} \sum_{i=1}^{n} \sum_{g=1}^{n}\left(A_{i 1} \cdot(g-1) \cdot\left[\left(\frac{g}{n}\right)^{m}-\left(\frac{g-1}{n}\right)^{m}\right]\right) .
$$




\section{Estimate for total average travel distance}

The total estimate for average travel distances in a warehouse with $n$ pick aisles, $k$ blocks, and $m$ picks can now be formulated as:

$$
T_{m}(n, k, y)=\sum_{i=1}^{11} E^{(i)}
$$

Note that equation 1 has been constructed to be as generally applicable as possible. This may make the equation overly complex for some special cases. For example, for $m=1$ we could simply estimate travel distances by $T_{1}(n, k, y)=y+k w_{c}+(n-1) w_{a}$ and for $k=1$ we could simplify our formulations to $T_{m}(n, 1, y)=E^{(1)}+E^{(2)}+2\left(E^{(4)}+E^{(5)}\right)$.

Furthermore, equation 1 is formulated for a fixed value of the number of picks $m$. Similar to Roodbergen and Vis (2006) we can easily adapt the estimates for a variable pick list size. Assuming that we know for every pick list size $m$ that it will occur with probability $p_{m}$ the average travel distance can be estimated as $T(n, k, y)=\sum_{m=1}^{\infty} p_{m} \cdot T_{m}(n, k, y)$. Finally, it might sometimes be interesting to study travel times instead of travel distances. The required translation can be made by dividing travel distances by the appropriate travel speed.

\section{$5 \quad$ Estimate quality and robustness}

In this section, we compare the travel distance estimate $T_{m}(n, k, y)$ from Section 4 with simulation. Furthermore, we test the quality of the solutions of the layout model of Section 2. We also investigate the quality of the layouts generated by the layout model if another routing policy is used for the actual operation of the warehouse than the S-shape policy we used to develop the distance estimate. Finally, we examine the quality of the solutions if another depot location is used.

We use a set of test instances, whose range covers the majority of practical warehouse layout optimization problems. We consider a manual picking operation, which may be in shelf racks, flow racks, pallet racks or any other type of racking. The main distinguishing factors between the various types of racking are the center-to-center distance between aisles $\left(w_{a}\right)$ and the cross aisle 
width $\left(w_{c}\right)$. Since these two factors are typically of the same order of magnitude, we will assume for all our experiments that they are identical $\left(w_{a}=w_{c}\right)$. Other important parameters for the layout optimization, as noted in Section 2, are the desired total aisle length $S$ and the number of picks per route $m$. For each of the three factors $w_{a}, S$, and $m$ we take the typical ranges as they occur in practice and select a set of evenly spaced points within the range. This gives us the following set of parameters for our test instances:

- $w_{a}=2,3,4,5,6$ meters;

- $S=100,200,300, \ldots, 800$ meters;

- $m=3,6,9,12, \ldots, 24$ picks.

This amounts to $5 \cdot 8 \cdot 8=320$ layout problems. For each of these 320 problems we determine the average travel distance with equation 1 of Section 4 and through simulation for 490 combinations of $n$ and $k$. This means that there are $320 \cdot 490=156,800$ instances to evaluate in total. For the simulation of each instance we run 2000 replications. Specifically, for each layout problem we investigate the following layouts:

- the number of aisles $(n)$ equals $2,3,4, \ldots, 50$;

- the number of blocks $(k)$ equals $1,2,3, \ldots, 10$.

\section{Comparison of the travel distance estimate with simulation}

Differences between the statistical estimate and simulation are calculated for all instances. Table 1 presents the results per value of the number of picks, because it has appeared from other research (Roodbergen and Vis, 2006), that estimate quality is more sensitive to changes in this parameter than to changes in the other parameters. Each row in the table contains the aggregate results of all evaluated instances for a given value of $m$. For example, the row for $m=15$ shows the maximum 
error encountered among 19,600 instances (all combinations of 5 values for $w_{a}, 8$ values for $S, 49$ values for $n$, and 10 values for $k$ ) and the average absolute estimation error over the same set of instances. The travel distances from the statistical estimate and from simulation are fairly close, as is apparent from Table 1 . The average absolute difference with simulation is $2.14 \%$ and the maximum absolute difference encountered among all instances was 7.96\%. There is no systematic bias that would allow for easy further improvement of the estimate. Of all evaluated instances the formula overestimated travel time in $55 \%$ of the cases when compared to simulated values, and underestimated travel time in $45 \%$ of the cases.

\section{XXXXXXXXXXXXXX}

\section{Insert table 1}

\section{XXXXXXXXXXXXXX}

\section{Comparison of layout optimizations with simulation}

To assess the performance of our optimization model of Section 2, we perform a layout optimization for each of the 320 layout problems in two ways. First, we determine the optimal layout according to the layout model of Section 2. Secondly, we determine the optimal layout through simulation. For the optimization through simulation we simply simulate all combinations of $n$ and $k$ and choose the one with the smallest average travel distance. We will refer to the two layouts as the "model's optimal layout" and the "simulated optimal layout" respectively. Next, for both layouts we determine the average travel distance through simulation. Note that we also determine the average travel distance for the model's optimal layout by means of simulation (instead of using the estimate's value). We do this to prevent a bias in our results. If we would determine the average travel distance for the model's optimal layout with the statistical estimate and for the simulated optimal layout through simulation, then differences between the two results could be caused by either a difference in the layout or by a difference in the travel distance estimate (we then could find differences even though the two layouts are identical). By determining average travel distances for both layouts through 
simulation we guarantee that if both approaches return the same layout, we will also find the same travel distance (the two results are simulated with the same random seed). Then we calculate the percentage difference between the travel distances resulting from the two layouts, which gives us an indicator for the quality of the layout optimization.

The results of the layout optimization comparisons are presented in column 'S-shape' of Table 2. The maximum error we encountered was $2.9 \%$. The average quality of the model is good with a deviation of only $0.3 \%$. An interesting point to note is that the quality of the layout optimization is actually better than the quality of the individual travel distance estimates, which we presented in Table 1. This is caused by the fact that there are several layouts that have a performance close to the optimal layout. For an illustration, see the example in the Appendix. Thus, the efficiency loss from selecting the wrong layout is fairly small, as long as the selected layout does not differ too much from the optimal layout. In $48 \%$ of the 320 instances we evaluated, the two optimization methods returned exactly the same layout and in $95 \%$ of the instances the difference between the two approaches was just one aisle and/or one block.

\section{$\operatorname{XXXXXXXXXXXXXX}$}

\section{Insert table 2}

\section{$\operatorname{XXXXXXXXXXXXXX~}$}

\section{Quality assessment for other routing methods}

In practice, the layout decision (a tactical decision) is often made before the decision concerning the routing policy (an operational decision) is made. Therefore it may be the case that a layout is chosen with our model, which is based on the assumption of the S-shape routing policy, but the actual operation will be using another routing method. To test the consequences of such action, we include results on two other routing policies in Table 2 .

A commonly used routing method is largest gap, which basically follows the perimeter of the blocks. Each subaisle of a block can be entered from one side or from both sides. Any subaisle is 
entered and left from the same cross aisle, except if a full traversal of a subaisle is needed to make a connection to the next block. Aisles are thus (1) entered and left from the back cross aisle, (2) entered and left from the front cross aisle, or (3) both. The shortest of the three options is chosen.

A routing method with a known good performance is the combined policy. With this policy, subaisles are visited in exactly the same sequence as with the S-shape policy. The combined policy, however, is capable of deciding to traverse a subaisle or to leave a subaisle from the same side it was entered. The decision to traverse or return in subaisles is optimized through dynamic programming. For detailed descriptions of both routing policies and a figure with example routes, refer to Roodbergen and De Koster (2001).

We follow similar steps as we did before to generate the S-shape column of Table 2. We optimize the 320 layout problems by means of the model, which is based on the S-shape routing policy. Then we optimize the layout by means of simulation using the largest gap (combined) routing policy. Finally, we compare the average travel distance in the model's optimal layout to the average travel distance in the simulated optimal layout, when using the largest gap (combined) routing policy in both layouts.

As can be seen from Table 2, the layout model also has a fairly good performance for the other two routing methods. On average the performance of the model's optimal layouts differs only by $0.9 \%$ from the simulated optimal layouts for largest gap and $2.4 \%$ for combined. Interestingly, this is in contrast with the findings in Roodbergen and Vis (2006), where large differences were found between optimizations with S-shape and largest gap for one-block layouts. Apparently, the added layout possibilities of having more than one block seem to stabilize the resulting layouts between the routing methods.

\section{Quality assessment for another depot location}

The formulas presented in this paper are based on the assumption that the depot is located in the front cross aisle. However, the depot may also be located at the head of a cross aisle (Caron et al., 
1998). This configuration is indicated in Figure 1 by "alternative depot location". We use the model without alterations for this test; the simulation explicitly uses the alternative depot location. The impact appears to be minor. The average difference in travel distances is $0.38 \%$ and the maximum difference is $4.1 \%$. Therefore, it can be concluded that the model is equally applicable to warehouses with another depot location.

\section{$6 \quad$ Layout experiments}

In this section, we investigate solutions of the layout optimization model to shed some light on how the variables $n$ (number of aisles) and $k$ (number of cross aisles) react to variations in the parameters $S$ (total aisle length), $m$ (number of picks), and $w_{a}$ (width of the aisles). As before we assume $w_{c}=w_{a}$ throughout all experiments. Very little is known from literature on these relations. Only Roodbergen and De Koster (2001) and Vaughan and Petersen (1999) study similar layouts. Both papers, however, essentially evaluate the effect of increasing the number of blocks for predefined values of the number of aisles. Here we allow simultaneous changes in both the number of aisles and the number of blocks.

We present the optimal layouts for several layout problems in Figure 4. These are the same layout problems as described in Section 5, but for ease of presentation instances with $w_{a}=3$ and $w_{a}=5$ have been omitted. The omitted instances, however, have been considered for the results as presented in Table 3. Figure 4 is organized as follows. For each value of $w_{a}\left(w_{a}=2,4,6\right)$ two $8 \times 8$ squares are given, positioned next to each other. The first square contains the optimal number of aisles $(n)$, and the second square contains the optimal number of blocks $(k)$. Each square consists of 64 cells ( 8 rows by 8 columns). Each cell contains the optimal value for a specific combination of $m$ and $S$ as indicated on the top $(S)$ and at the left $(m)$ of the squares.

\section{XXXXXXXXXXXXXX}

\section{Insert Figure 4}




\section{Insert Table 3}

\section{$\operatorname{XXXXXXXXXXXXXX}$}

Layout optimization essentially means finding the best balance between cross-aisle travel and within-aisle travel such that the total travel distance is minimized. If the aisles are very long, the insertion of an additional cross aisle will significantly reduce within-aisle travel and only slightly increase cross-aisle travel, which makes it a good choice. If the subaisles are fairly short, an extra cross aisle will still reduce within-aisle travel, but this gain will be smaller than the loss due to increased travel in the cross aisles. This trade-off was also noted as the main issue by Vaughan and Petersen (1999). The results from our experiments are consistent with these insights.

Based on our results, we can identify a number of probable relations between parameters $w_{a}$, $m, S$ and variables $n, k$. These relations are presented schematically in Table 3 . As can be seen from Table 3, larger areas (high $S$ ) require more subaisles, which can be achieved by increasing the number of aisles and/or the number of blocks. If the distance between aisles $\left(w_{a}\right)$ increases, then the cost of increasing the number of subaisles increases, which implies that fewer subaisles will be included in the optimal configuration.

From the 320 design problems we studied, only 56 of the optimal solutions consisted of just one block. Moreover, these 56 layouts all had 2 aisles in the optimum. These $n=2 / k=1$ solutions mainly appear when pick density is high and cross aisles are wide (i.e., aisle changing is costly). The remaining 264 optimal solutions all have multiple blocks. Thus, it seems that - apart from special cases in which the $n=2 / k=1$ is best - it is always better to have a multiple-block layout than a one-block layout.

\section{Concluding remarks}

In this paper, we developed a model that can be used to determine a layout structure for order picking areas in warehouses. Previous research restricted layouts to situations with only one block, 
essentially reducing the optimization problem to finding the best number of aisles. Also situations with two blocks were studied before, but the depot location was chosen in these studies such that the analysis could not be easily extended to more blocks. The optimization model presented in this paper is capable of considering layouts with any number of blocks and any number of aisles.

The objective function in the layout optimization model is formed by a statistical estimate for average travel distances in a warehouse with random storage and S-shape routing. Experiments show that the layouts generated by the model are generally similar to the layouts generated through simulation. Furthermore, travel distances in layouts optimized with our model are shown to differ on average by $0.3 \%$ and at most by $2.9 \%$ from travel distances in layouts obtained by simulation. Additional testing indicated that the layouts generated by the model are also fairly adequate if the actual operation of the warehouse will be using another routing method than the S-shape policy. Finally, the model was used to investigate the behavior of the optimal layout in response to changes in various parameters. Some probable relations have been identified between input parameters and optimal layout configurations.

\section{References}

Caron, F., Marchet, G. and Perego, A. (1998) Routing policies and COI-based storage policies in picker-to-part systems, International Journal of Production Research, 36(3), 713-732.

Caron, F., Marchet, G. and Perego, A. (2000) Optimal layout in low-level picker-to-part systems, International Journal of Production Research, 38(1), 101-118.

Chew, E.P. and Tang, L.C. (1999) Travel time analysis for general item location assignment in a rectangular warehouse, European Journal of Operational Research, 112, 582-597.

Gu, J., Goetschalckx, M. and McGinnis, L.F. (2007) Research on warehouse operation: a comprehensive review, European Journal of Operational Research, 177(1), 1-21.

Gue, K.R., Meller, R.D. and Skufca, J.D. (2006). The effects of pick density on order picking areas 
with narrow aisles, IIE Transactions 38(10), 859-868.

Hall, R.W. (1993) Distance approximations for routing manual pickers in a warehouse, IIE Transactions, 25(4), 76-87.

Jarvis, J.M. and McDowell, E.D. (1991) Optimal product layout in an order picking warehouse, IIE Transactions, 23(1), 93-102.

Kunder, R. and Gudehus, T. (1975) Mittlere Wegzeiten beim eindimensionalen Kommissionieren, Zeitschrift für Operations Research, 19, B53-B72.

Le-Duc, T. and De Koster, M.B.M. (2005) Travel distance estimation and storage zone optimization in a 2-block class-based storage strategy warehouse, International Journal of Production Research, 43(17), 3561-3581.

Petersen, C.G. (1997) An evaluation of order picking routing policies, International Journal of Operations 8 Production Management, 17(11), 1098-1111.

Ratliff, H.D. and Rosenthal, A.S. (1983) Orderpicking in a rectangular warehouse: a solvable case of the traveling salesman problem, Operations Research, 31(3), 507-521.

Roodbergen, K.J. and De Koster, R. (2001) Routing methods for warehouses with multiple cross aisles, International Journal of Production Research, 39(9), 1865-1883.

Roodbergen, K.J. and Vis, I.F.A. (2006) A model for warehouse layout, IIE Transactions, 38(10), 799-811.

Tompkins, J.A., White, J.A., Bozer, Y.A.. and Tanchoco, J.M.A. (2003) Facilities Planning, John Wiley and Sons, New York.

Vaughan, T.S. and Petersen, C.G. (1999) The effect of warehouse cross aisles on order picking efficiency, International Journal of Production Research, 37(4), 881-897. 


\begin{tabular}{|c|c|c|}
\hline picks $(\boldsymbol{m})$ & maximum error (\%) & average error (\%) \\
\hline $\mathbf{3}$ & 7.6 & 2.9 \\
\hline $\mathbf{6}$ & 8.0 & 3.7 \\
\hline $\mathbf{9}$ & 7.5 & 2.6 \\
\hline $\mathbf{1 2}$ & 4.8 & 2.0 \\
\hline $\mathbf{1 5}$ & 5.5 & 1.5 \\
\hline $\mathbf{1 8}$ & 5.8 & 1.4 \\
\hline $\mathbf{2 1}$ & 5.5 & 1.5 \\
\hline $\mathbf{2 4}$ & 5.6 & 1.6 \\
\hline
\end{tabular}

TABLE 1. Average and maximum of absolute deviations of the travel distance estimates from simulated values.

\begin{tabular}{|c|c|c|c|}
\hline & S-shape & Largest Gap & Combined \\
\hline average error (\%) & 0.3 & 0.9 & 2.4 \\
\hline maximum error (\%) & 2.9 & 3.7 & 6.4 \\
\hline
\end{tabular}

TABLE 2. Percentage difference between travel time in a layout determined with the model and a layout determined with routing-specific simulation for a test set of 320 layout problems.

\begin{tabular}{|l|c|c|}
\hline & $n$ & $k$ \\
\hline$W_{a}$ & $\downarrow$ & $\downarrow$ \\
\hline$S$ & $\uparrow$ & $\uparrow$ \\
\hline$m$ & $\downarrow$ & $\uparrow \downarrow$ \\
\hline
\end{tabular}

TABLE 3. Effect on the layout variables $n$ and $k$ of an upward change in one of the parameters $w_{a}, S$ or $m$. An upwards arrow means that the optimal value of the layout variable will increase (or at least remain equal) if the corresponding parameter increases. If both an upward and a downward arrow are given in a cell, then the variable may either increase, decrease or remain equal. Results are based on a set of 320 representative layout problems. 


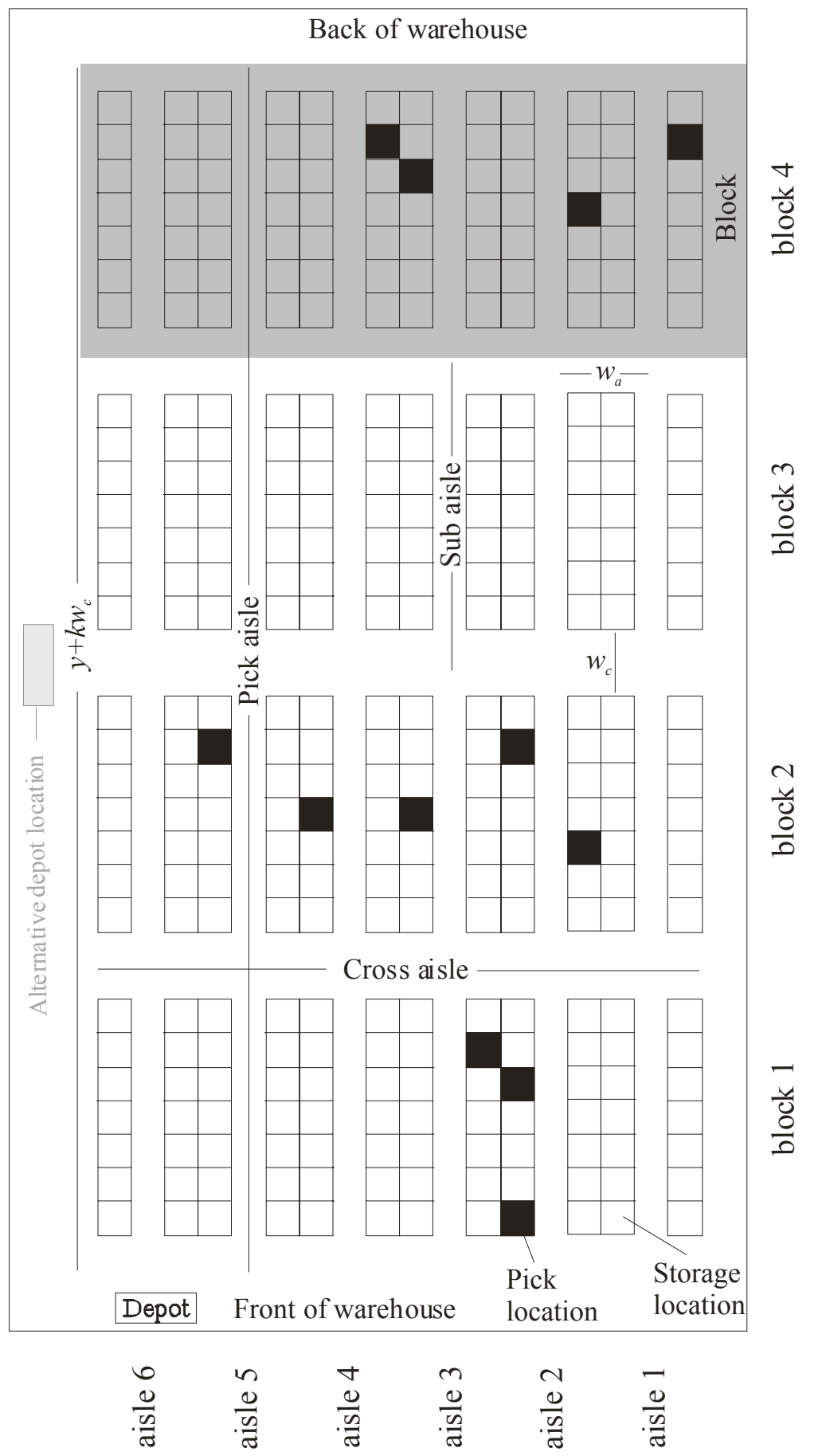

FIGURE 1. Schematic top view of a typical order picking area in a warehouse. 


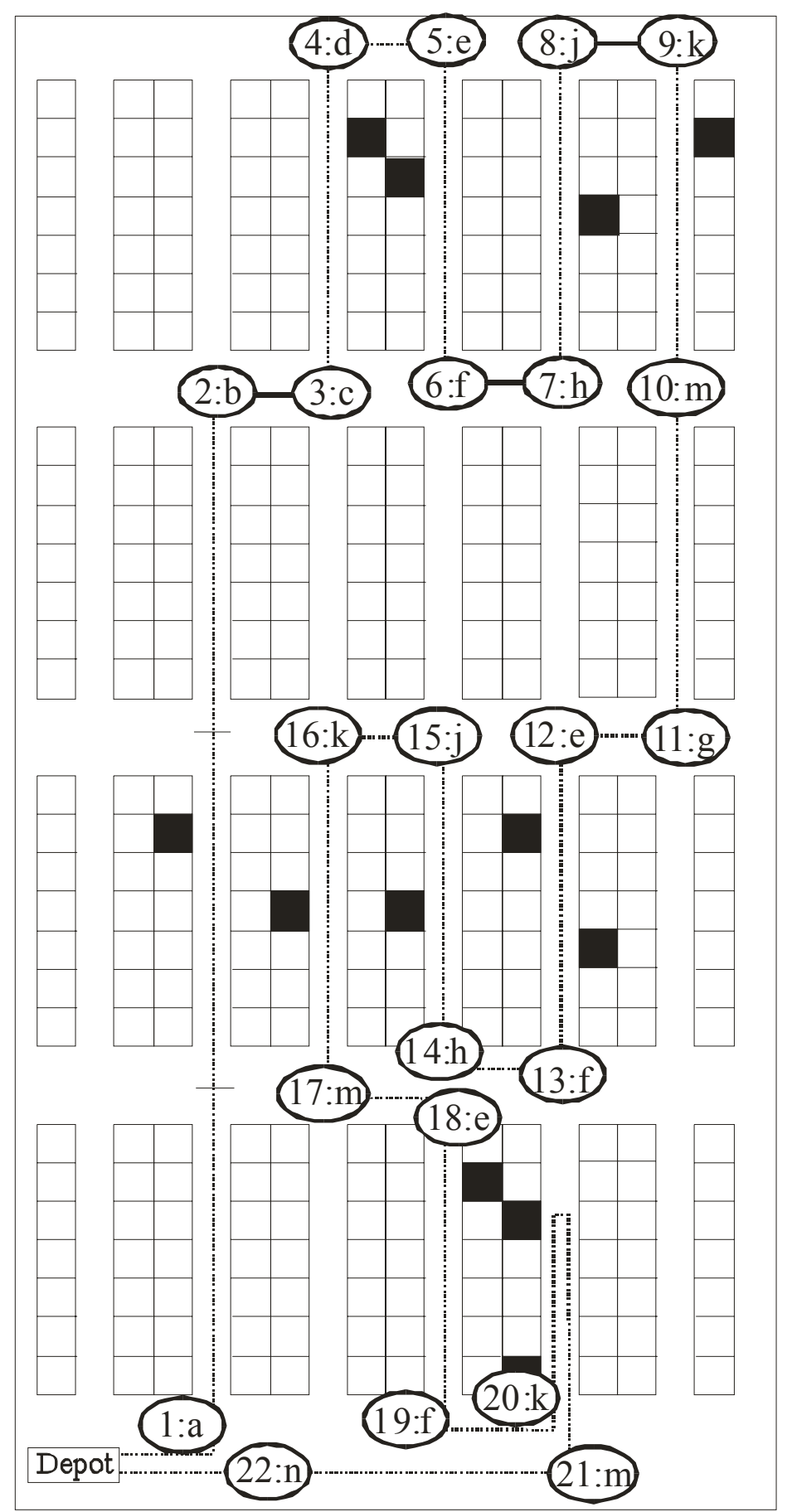

FIGURE 2. Example route as generated by the S-shape routing method. Numbers indicate the travel sequence. Letters in this figure correspond to the letters that are given in brackets in the description of the routing method in Section 3. 


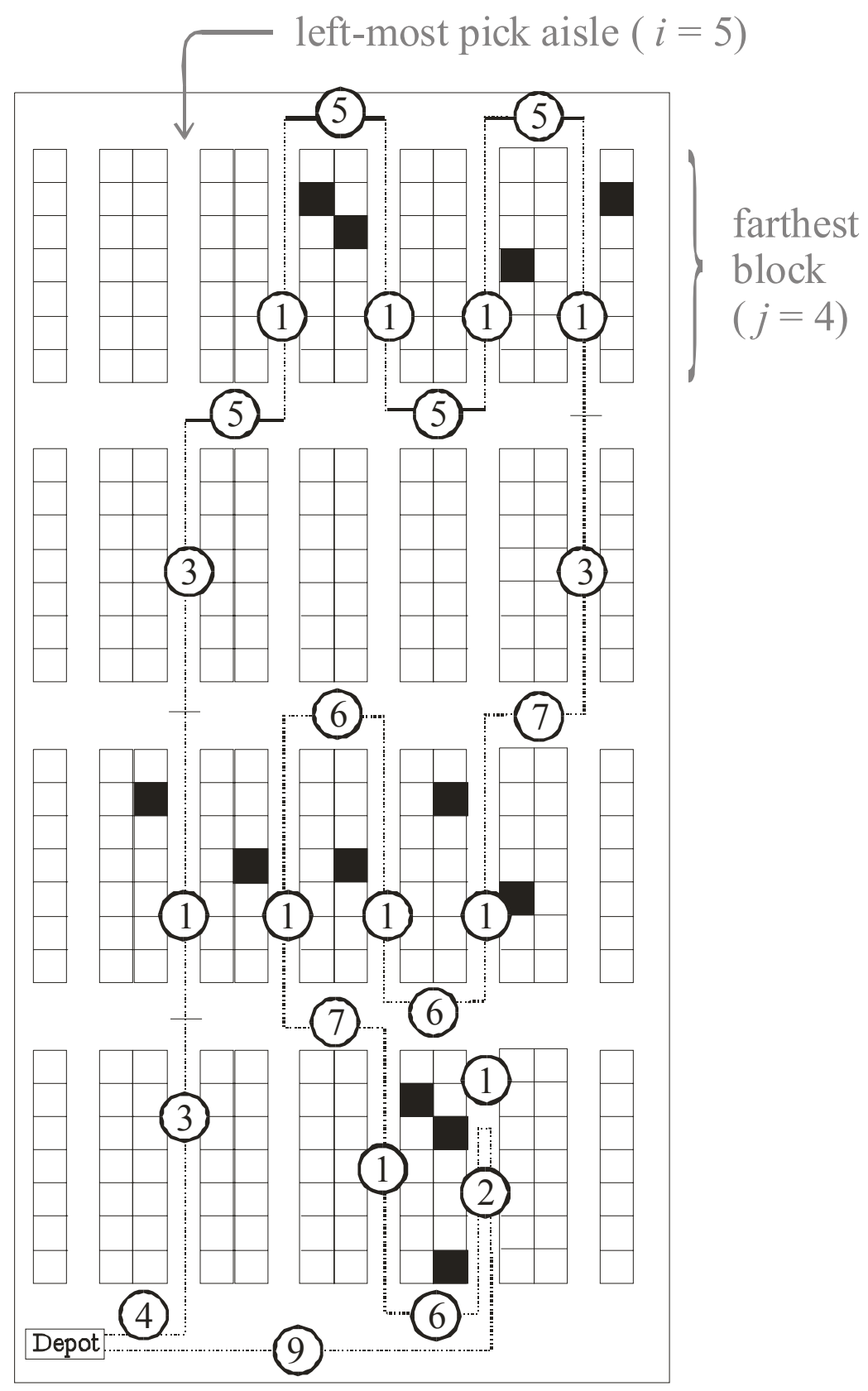

FIGURE 3. The same example route as in Figure 2. Numbers in this figure correspond to the numbers of the various components of the statistical estimate as described in Section 4. 


\begin{tabular}{|c|c|c|c|c|c|c|c|c|}
\hline $\mathrm{m} \backslash \mathrm{S}$ & 100 & 200 & 300 & 400 & 500 & 600 & 700 & 800 \\
\hline 3 & 7 & 10 & 12 & 14 & 16 & 17 & 18 & 20 \\
\hline 6 & 6 & 9 & 11 & 13 & 15 & 16 & 17 & 18 \\
\hline 9 & 6 & 9 & 10 & 12 & 13 & 14 & 16 & 17 \\
\hline 12 & 6 & 8 & 9 & 11 & 12 & 13 & 15 & 15 \\
\hline 15 & 5 & 7 & 9 & 10 & 11 & 13 & 13 & 14 \\
\hline 18 & 2 & 7 & 8 & 10 & 11 & 12 & 13 & 13 \\
\hline 21 & 2 & 6 & 8 & 9 & 10 & 11 & 12 & 13 \\
\hline 24 & 2 & 6 & 7 & 9 & 10 & 10 & 11 & 12 \\
\hline
\end{tabular}

optimal number of aisles $\left(w_{a}=2\right)$

\begin{tabular}{|c|c|c|c|c|c|c|c|c|}
\hline $\mathrm{m} \backslash \mathrm{S}$ & 100 & 200 & 300 & 400 & 500 & 600 & 700 & 800 \\
\hline 3 & 5 & 7 & 9 & 10 & 11 & 12 & 13 & 14 \\
\hline 6 & 4 & 6 & 8 & 9 & 10 & 11 & 12 & 13 \\
\hline 9 & 2 & 6 & 7 & 9 & 9 & 10 & 11 & 12 \\
\hline 12 & 2 & 6 & 6 & 8 & 9 & 9 & 10 & 11 \\
\hline 15 & 2 & 5 & 6 & 7 & 8 & 9 & 10 & 10 \\
\hline 18 & 2 & 2 & 6 & 7 & 7 & 8 & 9 & 10 \\
\hline 21 & 2 & 2 & 5 & 6 & 7 & 8 & 8 & 9 \\
\hline 24 & 2 & 2 & 2 & 6 & 7 & 7 & 8 & 9 \\
\hline
\end{tabular}

optimal number of aisles $\left(w_{a}=4\right)$

\begin{tabular}{|c|c|c|c|c|c|c|c|c|}
\hline $\mathrm{m} \backslash \mathrm{S}$ & 100 & 200 & 300 & 400 & 500 & 600 & 700 & 800 \\
\hline 3 & 4 & 6 & 7 & 8 & 9 & 10 & 11 & 12 \\
\hline 6 & 3 & 5 & 6 & 7 & 8 & 9 & 10 & 11 \\
\hline 9 & 2 & 4 & 6 & 7 & 8 & 9 & 9 & 10 \\
\hline 12 & 2 & 2 & 6 & 7 & 7 & 8 & 8 & 9 \\
\hline 15 & 2 & 2 & 5 & 6 & 7 & 7 & 8 & 8 \\
\hline 18 & 2 & 2 & 2 & 5 & 6 & 7 & 7 & 8 \\
\hline 21 & 2 & 2 & 2 & 2 & 6 & 6 & 7 & 8 \\
\hline 24 & 2 & 2 & 2 & 2 & 5 & 6 & 7 & 7 \\
\hline
\end{tabular}

optimal number of aisles $\left(w_{a}=6\right)$

\begin{tabular}{|c|c|c|c|c|c|c|c|c|}
\hline $\mathrm{m} \backslash \mathrm{S}$ & 100 & 200 & 300 & 400 & 500 & 600 & 700 & 800 \\
\hline 3 & 3 & 3 & 3 & 3 & 3 & 4 & 4 & 4 \\
\hline 6 & 4 & 4 & 5 & 5 & 5 & 5 & 6 & 6 \\
\hline 9 & 4 & 5 & 6 & 6 & 7 & 7 & 7 & 7 \\
\hline 12 & 4 & 6 & 7 & 7 & 8 & 8 & 8 & 9 \\
\hline 15 & 4 & 6 & 8 & 8 & 9 & 9 & 10 & 10 \\
\hline 18 & 1 & 6 & 9 & 9 & 9 & 10 & 10 & 11 \\
\hline 21 & 1 & 8 & 8 & 10 & 11 & 11 & 12 & 12 \\
\hline 24 & 1 & 8 & 10 & 10 & 11 & 13 & 13 & 13 \\
\hline
\end{tabular}

optimal number of blocks $\left(w_{a}=2\right)$

\begin{tabular}{|c|c|c|c|c|c|c|c|c|}
\hline $\mathrm{m} \backslash \mathrm{S}$ & 100 & 200 & 300 & 400 & 500 & 600 & 700 & 800 \\
\hline 3 & 2 & 3 & 3 & 3 & 3 & 3 & 3 & 3 \\
\hline 6 & 3 & 4 & 4 & 4 & 5 & 5 & 5 & 5 \\
\hline 9 & 1 & 4 & 5 & 5 & 6 & 6 & 6 & 6 \\
\hline 12 & 1 & 4 & 6 & 6 & 6 & 7 & 7 & 7 \\
\hline 15 & 1 & 4 & 6 & 6 & 7 & 8 & 8 & 8 \\
\hline 18 & 1 & 1 & 6 & 6 & 8 & 9 & 9 & 9 \\
\hline 21 & 1 & 1 & 6 & 8 & 8 & 8 & 10 & 10 \\
\hline 24 & 1 & 1 & 1 & 8 & 8 & 10 & 10 & 10 \\
\hline \multicolumn{10}{|c|}{0 timal number of blocks $\left(w_{a}=4\right)$} \\
\hline
\end{tabular}

\begin{tabular}{|c|c|c|c|c|c|c|c|c|}
\hline $\mathrm{m} \backslash \mathrm{S}$ & 100 & 200 & 300 & 400 & 500 & 600 & 700 & 800 \\
\hline 3 & 2 & 3 & 3 & 3 & 3 & 3 & 3 & 3 \\
\hline 6 & 2 & 3 & 4 & 4 & 4 & 4 & 5 & 5 \\
\hline 9 & 1 & 4 & 4 & 4 & 5 & 5 & 5 & 6 \\
\hline 12 & 1 & 1 & 4 & 4 & 6 & 6 & 7 & 7 \\
\hline 15 & 1 & 1 & 4 & 6 & 6 & 6 & 6 & 8 \\
\hline 18 & 1 & 1 & 1 & 6 & 6 & 6 & 8 & 8 \\
\hline 21 & 1 & 1 & 1 & 1 & 6 & 8 & 8 & 8 \\
\hline 24 & 1 & 1 & 1 & 1 & 8 & 8 & 8 & 10 \\
\hline \multicolumn{7}{|c|}{ optimal number of blocks $(w$} & $=6)$
\end{tabular}

optimal number of blocks $\left(w_{a}=6\right)$

FIGURE 4. Overview of the optimal number of aisles $(n)$, and the optimal number of blocks $(k)$ for a series of layout problems. The row of a square indicates the number of picks $(m)$, the column of a square indicates the total aisle length $(S)$. 


\section{Appendix: Example of a layout optimization}

We consider a layout problem with $w_{a}=4, w_{c}=4, S=300$ and $m=9$. We calculate the average travel distance for all values of $n=2, \ldots, 50$ and $k=1, \ldots, 10$ with equation (1). Aisle length is determined as $y=S / n$. Figure A.1 shows 10 curves; one for each value of the number of blocks $(k)$. Each curve gives the estimated average travel distance as a function of the number of aisles $(n)$. Especially, the curve for $k=1$ has a significantly different shape than the other curves. This can be explained as follows. The curve starts at a layout with two aisles $(n=2)$, in which almost all routes will go up aisle 1 and down aisle 2 . The second point on the $k=1$ curve consists of a layout with three aisles $(n=3)$. Since we have an expected value of three picks per aisle $(m / n=3)$, there is a large probability of visiting all three aisles and thus of having to make a turn in the third aisle. The expected length of a turn is equal to the length of an aisle only if there is one pick in the aisle. With an expected number of three picks per aisle, we can expect to travel 1.5 times the aisle length in the third aisle (see estimate $E^{(2)}$ ). This extra distance in the third aisle explains the peak at $n=3$. The next point on the curve is $n=4$ which will mainly have routes without turns. Then the next point, $n=5$, again has a significant probability of having turns in the last aisle. For $n=5$, however, the actual extra travel will be lower since the expected number of picks per aisle is just 1.8, which causes a smaller peak.

\section{XXXXXXXXXXXXXX}

\section{Insert Figure A.1}

\section{XXXXXXXXXXXXXX}

The best layout for this example is at $n=7, k=5$. Looking at the curves in Figure A.1, we can see that there are many curves with similar travel distances around the minimum. Furthermore, all curves appear relatively flat around the optimum. To investigate this, we created Figure A.2. The horizontal axis of this figure gives the number of blocks and the 
vertical axis gives the number of aisles. Thus, the position of a dot in the figure represents a specific layout. The optimal layout has been marked with a circle. All layouts that have an average travel distance that differs at most $1 \%$ from the optimal layout are indicated with a white square. All layouts differing more than $1 \%$, but no more than $3 \%$ are indicated with a grey square. Finally, black squares indicate layouts that have travel distances which differ between $3 \%$ and $5 \%$ from the travel distances in the optimal layout. The figure clearly shows that there are many layouts with a performance that differs only a few percent from the optimal layout. Furthermore, other good layouts seem to be similar to the optimal layout, with only one or a few aisles or blocks more or less. One interesting point in Figure A.2 is the fact that no dots occur for layouts with 3 blocks. This can be explained as follows. A typical route in a three-block layout visits the aisles in the block farthest from the depot from left to right. In the next block, aisles are typically visited from right to left. Finally, in the block closest to the depot, aisle are visited left to right. This means that routes tend to be on the right of the front cross aisle after picking the last item, while the depot is at the left. Thus the order picker must traverse a large part of the front cross aisle without picking in layouts with three blocks. This makes the three-block layout likely to be less efficient than a layout with 2 or 4 blocks.

\section{XXXXXXXXXXXXXX}

Insert Figure A.2

XXXXXXXXXXXXXX 


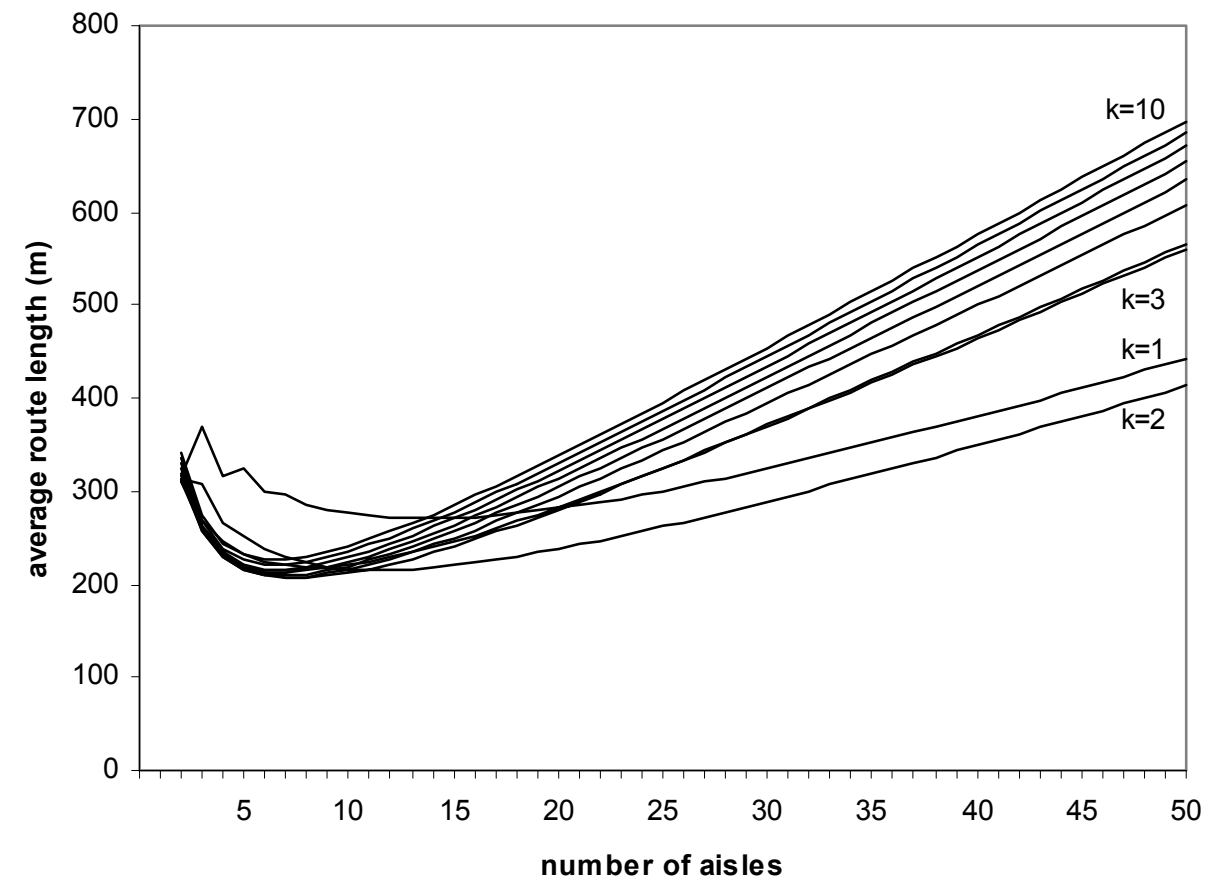

Figure A.1. Average travel time as a function of the number of aisles for a layout optimization problem with $w a=4, S=300, m=9$. Each curve corresponds to a situation with a given number of blocks (as indicated next to the curves). 


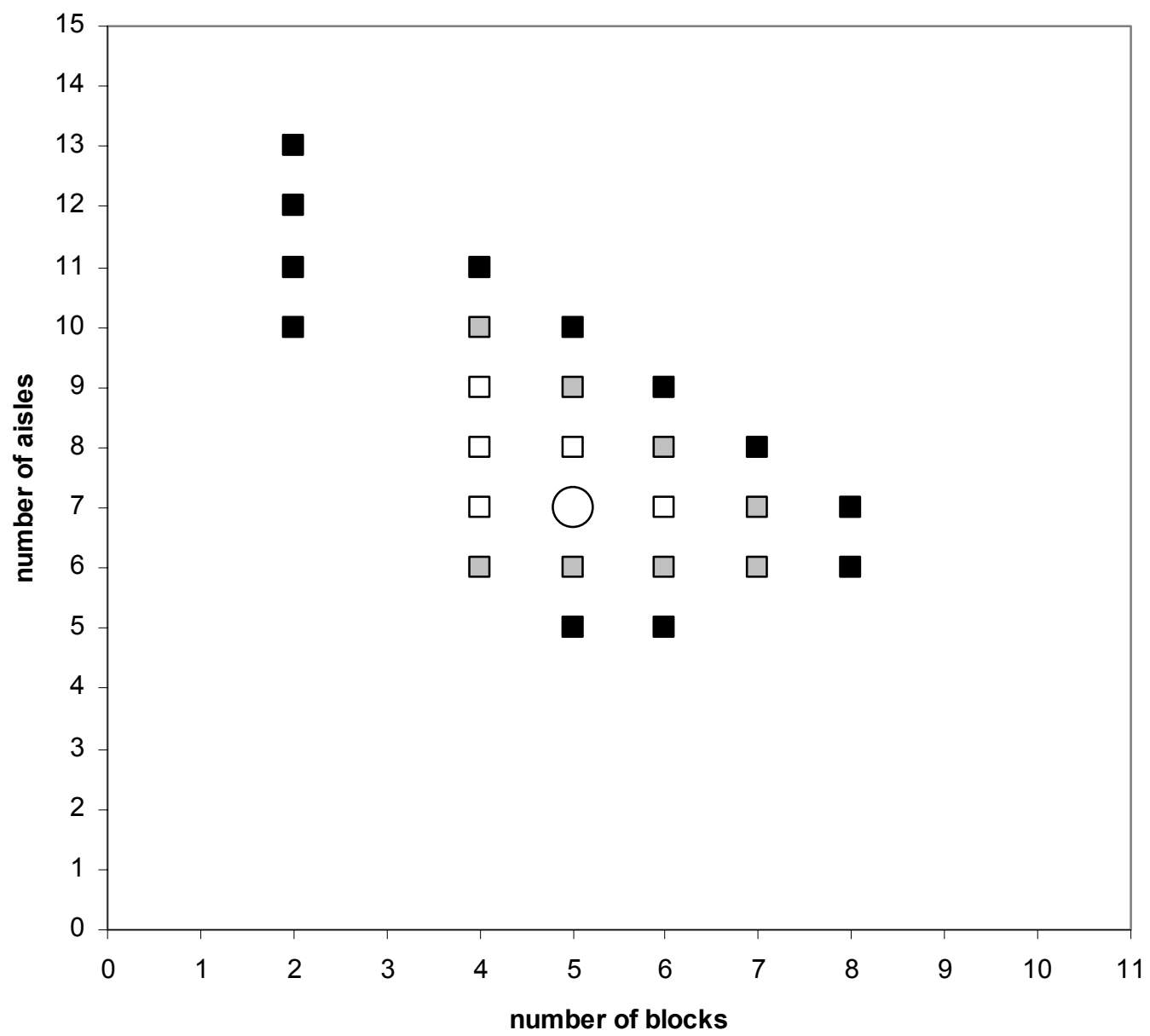

Figure A.2. The optimal layout (indicated with a circle) and several other layouts for a layout optimization problem with $w_{a}=4, S=300, m=9$. White squares indicate layouts that differ at most $1 \%$ from the optimal layout. Layouts indicated with grey squares differ at most 3\% from the optimum, and layouts indicated with black squares differ up to $5 \%$ from the optimum. 